\title{
Some perceptual dependencies in speeded classification of vowel color and pitch
}

\author{
THOMAS D. CARRELL, LINDA B. SMITH, and DAVID B. PISONI \\ Indiana University, Bloomington, Indiana 47405
}

\begin{abstract}
Wood (1975) suggested that information specifying consonant identity is dependent on the earlier processing of pitch. Is vowel identification also dependent on the prior processing of pitch? In contrast with the results obtained with consonants, Kuhl $(1975,1976)$ reported that infants responded selectively to differences in vowel color when pitch varied but not to differences in pitch when vowel color varied. Miller (1978) has also reported that adults show mutual, symmetric interference effects in speeded classification of vowel color and pitch. In the present study, judgments of vowel color and pitch were examined in a reaction time task in order to assess the effects of the relative discriminability of vowel and pitch quality on speeded classification. In addition, we also compared the classification of isolated vowels to vowels in consonantal context. The overall results were consistent with both Kuhl's and Miller's earlier findings but refine our understanding of the interaction between various dimensions by showing that vowel identification is also dependent on the processing of pitch information. Such an interaction, however, becomes evident only when the processing dependencies are examined across a wide range of stimulus values for each dimension. The present findings provide additional information about the nature of processing dependencies among dimensions in speech and the methods by which such dependencies may be studied.
\end{abstract}

Nearly all work in speech perception is based on the assumption that several stages of processing interact to decode the acoustic waveform into a sequence of linguistic units (see Pisoni, 1978; Studdert-Kennedy, 1976). The precise nature of these processing stages is not clear at the present time, but the existence of distinct auditory and phonetic levels of perceptual processing has been widely hypothesized in the literature on speech perception. The basic notion is that the auditory level operates on all acoustic information, whereas the phonetic level operates only on information specific to the analysis of speech into phonetic segments and features (see, e.g., Studdert-Kennedy, 1976).

Wood and his associates have attempted to distinguish these two levels of processing via a speeded selective attention task (Day \& Wood, 1972; Wood, 1975; Wood, Goff, \& Day, 1971). In one experiment, Wood (1975) presented subjects with synthetic speech sounds that varied on a general auditory dimension (pitch) and a more specific phonetic dimension (place of articulation). The subjects were asked to classify the sounds according to one or the other dimension. Wood found that subjects were able to classify synthetic syllables according to pitch unaffected by orthogonal variation in place of articulation. However, the subjects' classification of the stimulus according to place of articulation was slowed down by the addition of pitch variation. This pattern of results is consistent

This research was supported by NIMH Research Grant MH. 24027-05, NIH Grant NS-12179-04, and NSF Grant BNS 78-13019 to Indiana University in Bloomington. with two levels of processing. Asymmetric dependencies were assumed to exist between place of articulation and pitch because the processing of place was thought to occur at a later phonetic stage of processing that is dependent on the earlier auditory level at which pitch is processed.

By this analysis, the processing of general auditory dimensions such as pitch or loudness should be independent of, and prior to, the processing of all types of phonemic information in speech. However, there are several reasons to suspect that this pattern of dependencies might not be observed when the phonemes are vowels and the dimensions to be compared are other auditory attributes such as pitch or loudness. The acoustic cues specifying vowels in English are very different from those specifying stop consonants. Yowels have a much slower spectral rate of change than do stop consonants. And vowels and consonants have been shown to be encoded differently in various short-term memory tasks. For example, the immediate recall of vowels shows modality, recency, and suffix effects, whereas recall of consonants does not (Crowder, 1971; Crowder, 1973a, 1973b). In addition, it has often been reported that vowels tend to be perceived continuously while stop consonants are perceived more categorically, although this outcome depends largely on the manner in which the tests for categorical perception are carried out (Pisoni, 1971, 1973, 1975).

Recently, Miller (1978) reported results suggesting that the processing dependencies between vowel and pitch are, in fact, different from those found by Wood (1975) for processing place of articulation of stop 
consonants and pitch. Miller's task was essentially the same as that used by Wood (1975), except that the stimuli were $\mathrm{CV}$ syllables that varied in vowel color and pitch. Miller found a mutual, symmetric interference effect when these stimulus dimensions varied. Specifically, when the subject's task was vowel identification, classification of the sounds according to the vowel category was slowed down by the addition of the pitch variation. When the subject's task was to classify the stimuli according to pitch, performance was also slowed down by the addition of vowel variation. Moreover, the magnitude of the interference effect did not differ between the vowel identification and pitch identification tasks. The overall pattern of results observed by Miller is consistent with the notion that vowels are processed at the same level of perceptual analysis as pitch-a level that corresponds to a global analysis of the auditory qualities of the stimuli in terms of overall similarity in the absence of any phonetic recoding into distinctive features.

Kuhl (1976) has also reported findings pertinent to the nature of the interaction between the processing of pitch and vowel color. Using a sucking-habituation paradigm, Kuhl found that infants' discrimination of vowel color was unaffected by variation in pitch. In contrast, infants' discrimination of pitch was disrupted by variations in vowel color. It should be noted that an asymmetric pattern of interference such as the one reported by Kuhl is precisely the opposite of that observed by Wood between pitch and consonant classification and by Miller for vowel and pitch. These results raise the question of how to interpret asymmetric interference effects in speech-perception experiments of this kind. Certainly, one would not want to conclude, as Kuhl has, that infants' processing of vowels is prior to their discrimination of pitch. In other words, asymmetric interference effects may result from factors other than processing dependencies per se.

One possible account of the observed asymmetries is that the two stimulus dimensions simply differ in their inherent discriminabilities (see Garner \& Morton, 1969). If one dimension is considerably more discriminable than the other, identification of values on the more discriminable dimension may not be measurably affected by the small variation on the second dimension. This outcome would be anticipated simply because it is easier to ignore small irrelevant differences that are difficult to discriminate. However, identification of the values on the less discriminable dimension may be substantially affected by variation on the more discriminable dimension because it is difficult to ignore large stimulus differences. Differences in the discriminability between the two dimensions could then easily account for Kuhl's results with infants because the discriminability of the two dimensions was not measured or equated in her study. However, the results reported by Wood and Miller are not subject to this interpretation. In both of these studies, the discrimi- nability of the two dimensions was measured and did not differ from each other. Still, the dependence observed in these studies need not reflect different levels of processing. As Kuhl (1976) has suggested, asymmetric dependencies may result from intrinsic differences in the "salience" of the two dimensions that are independent of differences in their discriminability. This is the account she offered for her infant findings, although she never systematically explored the question of how to define salience.

Even if we take Wood's and Miller's results as indicating real processing dependencies, there is still an important issue that is unresolved, and that is the scope of the dependency. Real processing dependencies may exist between specific values on particular dimensions or between certain dimensions without there being distinct differences between a general and phonetic level "of analysis in speech perception. In particular, certain values of pitch may interact with the identification of certain classes of phonemes even though there may be only one level of auditory analysis, albeit a relatively complex one. In contrast, the existence of a general auditory level of analysis that precedes a more specific stage of phonetic analysis in speech perception implies dependency effects across ranges of discriminabilities and ranges of specific stimulus values. If the perception of speech is truly secondary to, and dependent on, a prior nonspecific level of auditory processing, the perception of speech sounds should be more affected by manipulations of auditory dimensions than the other way around and such a dependency relation should be evident across a variety of specific stimulus values.

In the present experiments, we investigated the claims about an auditory and a phonetic level of processing by examining the dependencies between the processing of pitch and vowel color over a wide range of pitch values and vowel values. The specific question we addressed was whether manipulations of the discriminability of pitch would have a greater effect on vowel identification than would manipulations of the discriminability of vowels on pitch identification. It was expected that increases in the magnitude of irrelevant variation would lead to increases in the interference effect in both cases. However, the critical issue was whether the magnitude of the interference effect would depend on the discriminability of the irrelevant dimension more in one case than in the other. Specifically, would vowel identification be affected to a greater degree by increases in the discriminability of pitch variation than would pitch identification be affected by increases in the discriminability of vowel variation? The answer should be positive if vowel identification depended on the prior processing of pitch.

The processing of pitch and isolated vowels was examined in Experiment 1, and the processing of pitch and vowels in the context of a CV syllable was exam- 
Table 1

Parameter Values for Vowel Stimuli Used in Experiments 1 and 2

\begin{tabular}{|c|c|c|c|c|c|c|}
\hline $\begin{array}{c}\text { Vowel } \\
\text { Number }\end{array}$ & $\begin{array}{c}\text { IPA } \\
\text { Symbol }\end{array}$ & $\begin{array}{c}\text { Alphanumeric } \\
\text { Label }\end{array}$ & $\begin{array}{c}\text { Pitch } \\
\text { Contour }\end{array}$ & F1 & F2 & F3 \\
\hline 1 & /i/ & $\begin{array}{l}\text { EE1 } \\
\text { EE2 } \\
\text { EE3 }\end{array}$ & $\begin{array}{c}145-116 \\
130-104 \\
70-56\end{array}$ & $\begin{array}{l}270 \\
270 \\
270\end{array}$ & $\begin{array}{l}2290 \\
2290 \\
2290\end{array}$ & $\begin{array}{l}3010 \\
3010 \\
3010\end{array}$ \\
\hline 3 & 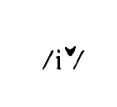 & $\begin{array}{l}\text { EEL1 } \\
\text { EEL2 } \\
\text { EEL3 }\end{array}$ & $\begin{array}{c}145-116 \\
130-104 \\
70-56\end{array}$ & $\begin{array}{l}298 \\
298 \\
298\end{array}$ & $\begin{array}{l}2226 \\
2226 \\
2226\end{array}$ & $\begin{array}{l}2902 \\
2902 \\
2902\end{array}$ \\
\hline 6 & $/ I^{\wedge} /$ & $\begin{array}{l}\text { IHH1 } \\
\text { IHH2 } \\
\text { IHH } 3\end{array}$ & $\begin{array}{c}145-116 \\
130-104 \\
70-56\end{array}$ & $\begin{array}{l}353 \\
353 \\
353\end{array}$ & $\begin{array}{l}2103 \\
2103 \\
2103\end{array}$ & $\begin{array}{l}2719 \\
2719 \\
2719\end{array}$ \\
\hline 7 & $/ \mathrm{I} /$ & $\begin{array}{l}\text { IH1 } \\
\text { IH2 } \\
\text { IH3 }\end{array}$ & $\begin{array}{c}145-116 \\
130-104 \\
70-56\end{array}$ & $\begin{array}{l}374 \\
374 \\
374\end{array}$ & $\begin{array}{l}2070 \\
2070 \\
2070\end{array}$ & $\begin{array}{l}2666 \\
2666 \\
2666\end{array}$ \\
\hline 13 & $|\epsilon|$ & $\begin{array}{l}\text { EH1 } \\
\text { EH2 } \\
\text { EH3 }\end{array}$ & $\begin{array}{c}145-116 \\
130-104 \\
70-56\end{array}$ & $\begin{array}{l}530 \\
530 \\
530\end{array}$ & $\begin{array}{l}1840 \\
1840 \\
1840\end{array}$ & $\begin{array}{l}2480 \\
2480 \\
2480\end{array}$ \\
\hline
\end{tabular}

ined in Experiment 2. The reason for this comparison was the possibility of greater involvement of the hypothesized secondary level of phonetic analysis when vowels are presented in dynamic phonetic context than when they are presented in isolation.

\section{EXPERIMENT 1}

\section{Method}

Sabjects. Seventy-eight students attending Indiana University were paid $\$ 3 / \mathrm{h}$ or were given experimental credit as part of a course requirement for their participation in this experiment. All subjects were native speakers of English, and none of the subjects reported a previous history of speech or hearing disorders.

Stimull. The stimuli consisted of 15 unique sounds, five vowels at three possible pitches. Differences in vowel quality were based on a stimulus continuum, generated earlier by Pisoni (1971), that ranged perceptually from $/ i /$ to $/ \mathrm{l} /$ to $/ \varepsilon /$ in 13 approximately equal logarithmic steps. In this continuum $/ i$ / is vowel number $1, / 1 /$ is vowel number 7 , and $/ \varepsilon /$ is vowel number 13 . Vowels between these endpoints also fall perceptually between $/ \mathrm{i} /, / \mathrm{I} /$, and $/ \varepsilon /$. The vowels chosen for the present experiment were stimulus numbers $1,3,6,7$, and 13 from this original series. Vowels 1 and 13 were used in the "large-vowel-difference" conditions, vowels 1 and 7 were used in the "intermediate-voweldifference" conditions, and vowels 3 and 6 were used in the "smallvowel-difference" conditions. Vowel 3 is generally identified as an $/ \mathrm{i} /$ and vowel 6 is generally identified as an / $/$ by naive subjects (Pisoni, 1971). The exact formant frequencies of these vowels are shown in Table 1.

The F0 of each vowel started at one of three possible frequencies: 145,130 , or $70 \mathrm{~Hz}$. In each case, the F0 dropped $25 \%$ linearly from the onset to the offset of the vowel. This pitch contour was implemented merely to increase the naturalness of the speech stimuli. The exact values for the F0 of each vowel are also specified in Table 1 . The three pitches are numbered 1 to 3 , from highest to lowest. The contrast between pitch 1 and pitch 3 , a difference of $75 \mathrm{~Hz}$, was used in the "large-pitch-difference" conditions and the contrast between pitch 1 and pitch 2, a difference of $15 \mathrm{~Hz}$, was used in the "small-pitch-difference" conditions.

Each vowel was $150 \mathrm{msec}$ in duration and had rise and fall times of $20 \mathrm{msec}$. Stimuli were presented at approximately $80 \mathrm{~dB}$ SPL on TDH-39 headphones after being low-pass filtered at $4.9 \mathrm{kHz}$. The vowels were synthesized on a version of the Klatt software synthesizer, outputing at a $10,000-\mathrm{Hz}$ sampling rate (Kewley-Port, 1978) as implemented on a PDP-11/05 in the
Speech Perception Laboratory at Indiana University in Bloomington. All stimuli were equated on the other parameters, except for those specifically varied for this experiment.

Procedure. Five experimental conditions were presented to five different groups of subjects. Each group of subjects participated in only one condition. The conditions may be characterized by the degree of discriminability of the values of pitch and vowel color as follows: (A) large vowel difference, small pitch difference; (B) intermediate vowel difference, small pitch difference; (C) large vowel difference, large pitch difference; (D) small vowel difference, small pitch difference; and $(E)$ small vowel difference, large pitch difference.

Subjects in each of the five conditions completed six classification tasks: two pitch-identification control tasks, one pitchidentification focusing task, two vowel-identification control tasks, and one vowel-identification focusing task. In the control tasks, the two stimuli differed only on the relevant dimension (vowel or pitch). The irrelevant dimension was fixed at one level for the first control task and at the second level for the second control task. In the focusing tasks, the four stimuli were composed of all combinations of two values of vowel and the two values of pitch. The resulting six identification tasks that were presented to each subject are summarized in Table 2.

The actual values of levels 1 and 2 depended on which of the five conditions a particular group of subjects was assigned to. For example, in the large-vowel-difference/small-pitch-difference condition, the vowels used were: EE1, EE2, EH1, and EH2. However, in the large-vowel-difference/large-pitch-difference condition, the vowels used were: EE1, EE3, EH1, and EH3 (see Table 1).

Each task consisted of 64 trials. The stimuli for each task were presented equally often in a pseudorandom order. The ordering of the tasks was counterbalanced in accordance with a Latin

Table 2

Arrangement of Stimuli for Each Block

\begin{tabular}{cccc}
$\begin{array}{c}\text { Target } \\
\text { Dimension }\end{array}$ & Control 1 & Control 2 & Focusing \\
\hline \multirow{2}{*}{ Vowel } & $\mathrm{V}_{1} \mathrm{P}_{1}$ & $\mathrm{~V}_{1} \mathrm{P}_{2}$ & $\mathrm{~V}_{1} \mathrm{P}_{1}$ \\
& $\mathrm{~V}_{2} \mathrm{P}_{1}$ & $\mathrm{~V}_{2} \mathrm{P}_{2}$ & $\mathrm{~V}_{2} \mathrm{P}_{1}$ \\
& & & $\mathrm{~V}_{1} \mathrm{P}_{2}$ \\
& & $\mathrm{~V}_{2} \mathrm{P}_{1}$ \\
& $\mathrm{~V}_{1} \mathrm{P}_{1}$ & $\mathrm{~V}_{2} \mathrm{P}_{1}$ & $\mathrm{~V}_{1} \mathrm{P}_{1}$ \\
& $\mathrm{~V}_{1} \mathrm{P}_{2}$ & $\mathrm{~V}_{2} \mathrm{P}_{2}$ & $\mathrm{~V}_{2} \mathrm{P}_{1}$ \\
& & & $\mathrm{~V}_{1} \mathrm{P}_{2}$ \\
& & & $\mathrm{~V}_{2} \mathrm{P}_{2}$ \\
\hline
\end{tabular}


A

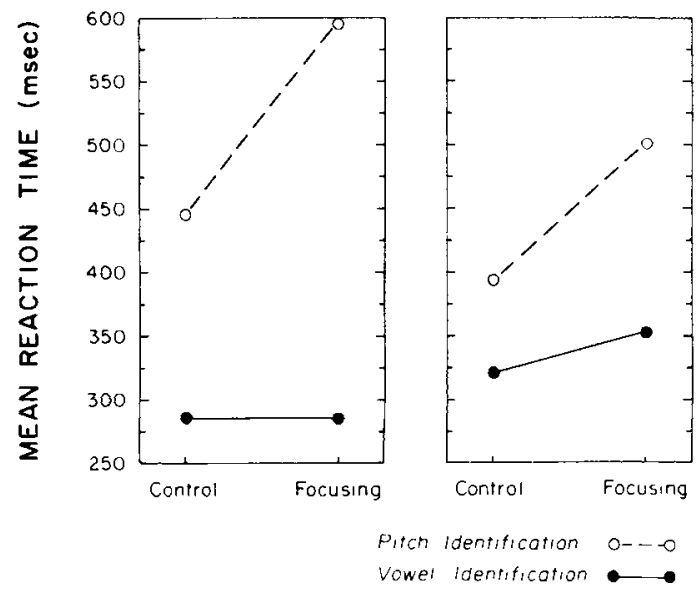

C

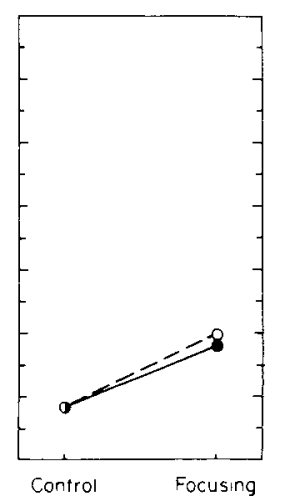

D

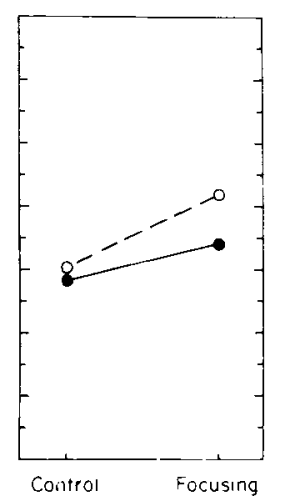

E

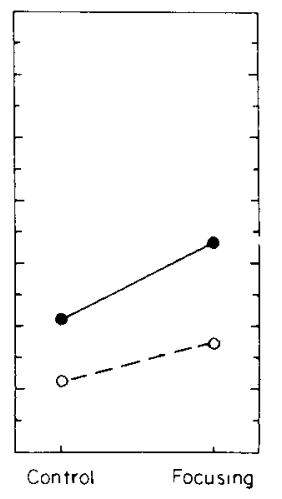

Figure 1. Mean reaction times for large-vowel/small-pitch, intermediate-vowel/small-pitch, large-vowel/large-pitch, small-vowel/ small-pitch, and small-vowel/large-pitch conditions in Experiment 1.

square design. All experimental events were controlled on-line by a PDP-11/05 computer.

At the beginning of the experiment, the subjects were given general instructions which included information that the trials would be self-paced and that the more quickly they responded, the sooner they would be finished. At the beginning of each task, the subjects were informed of the relevant dimension and again encouraged to respond as rapidly and as accurately as possible.

The entire session, consisting of instructions and the six tasks of 64 trials each, took from $\mathbf{4 0}$ to $50 \mathrm{~min}$. Pilot data, which were divided into quartiles, had indicated that reaction times reached asymptotic levels within the first quartile. Therefore, the first 16 trials in each block were considered as practice trials and were not analyzed further.

\section{Results and Discussion}

Mean reaction times in the five conditions are shown in Figure 1, and the specific values are given in Table 3. These means are based on correct responses only. Responses that took over $1.75 \mathrm{sec}$ (which constituted fewer than $1 \%$ of the trials) were counted as errors and not included in the data analysis. The two unique control blocks for each control task did not differ significantly, and reaction times in these two blocks were collapsed.

A three-way factorial analysis of variance was carried out on the reaction times in each condition (Subject by Task by Dimension). The two levels of task were control and focusing; the two levels of the dimension to be identified were vowel color and pitch. In some cases, repeated-measures $t$ tests were used to determine if there was a reliable difference between individual means. Error scores were also calculated from the data. The overall error rate averaged $5 \%$. Analyses of the error scores taken alone yielded essentially the same results as the analyses of the reaction time data, and therefore the error data will not be considered further.

Panel A in Figure 1 illustrates the pattern of reaction times when subjects identified stimuli that varied greatly in vowel color but only minimally in pitch. Examination of the figure shows that pitch identification was much slower than vowel identification $[F(1,11)=29.67, p<.0002]$. In addition, irrelevant vowel variation impaired pitch identification, but irrelevant pitch variation did not affect vowel identification. Specifically, only in the pitch identification task was performance in the focusing task reliably slower than in the control task $[F(1,11)=12.95, p<.004]$.

Mean reaction times for the condition of an intermediate vowel difference and a small pitch difference are illustrated in Panel B of Figure 1. Pitch and vowel discriminability are closer in this condition, as evidenced by the smaller difference in the control reac-

Table 3

Mean Reaction Times (in Milliseconds) for All Conditions in Experiment 1

\begin{tabular}{|c|c|c|c|c|c|c|c|c|c|c|}
\hline & \multicolumn{2}{|c|}{$\begin{array}{l}\text { Large Vowel, } \\
\text { Small Pitch }\end{array}$} & \multicolumn{2}{|c|}{$\begin{array}{l}\text { Intermediate Vowel, } \\
\text { Small Pitch }\end{array}$} & \multicolumn{2}{|c|}{$\begin{array}{l}\text { Large Vowel, } \\
\text { Large Pitch }\end{array}$} & \multicolumn{2}{|c|}{$\begin{array}{c}\text { Small Vowel, } \\
\text { Small Pitch }\end{array}$} & \multicolumn{2}{|c|}{$\begin{array}{l}\text { Small Vowel, } \\
\text { Large Pitch }\end{array}$} \\
\hline & $P$ & V & $\mathbf{P}$ & V & $\mathbf{P}$ & V & $\mathbf{P}$ & V & $\mathbf{P}$ & V \\
\hline Control & 445 & 284 & 393 & 320 & 292 & 292 & 402 & 391 & 305 & 354 \\
\hline Focusing & 595 & 284 & 500 & 352 & 348 & 340 & 458 & 420 & 337 & 415 \\
\hline
\end{tabular}

Note $-P=$ pitch $I D ; V=$ vowel $I D$. 
tion times. However, an asymmetric pattern of interference was still obtained, as indicated by the interaction between task and dimension $[F(1,17)=6.33$, $p<.02$ ]. Specifically, the interference effect (i.e., the difference between the focusing and control conditions) was greater in the pitch-identification task than in the vowel-identification task $[t(17)=2.48$, $\mathrm{p}<.02]$.

The results of the large-vowel-difference/large pitch difference condition are illustrated in Panel C. In this condition, a mutual symmetric pattern of interferences was observed. Specifically, performance in the two control tasks did not differ $[t(11)<$ 1.00 ], suggesting that the pitches and vowels were equally discriminable in this condition. Second, variation of the irrelevant dimension interfered with the identification of the relevant dimension $[F(1,11)=$ $13.37, p<.004$ ], but there was no interaction between task and dimension $[F(1,11)<1.00]$.

Performance in the condition in which there was a small vowel and a small pitch difference is shown in Panel D. Again, performance in the two control tasks did not differ $[t(17)<1.00]$, suggesting that the specific pitches and vowels were equally discriminable, a pattern of results implicating mutual and symmetric dependencies between the two dimensions. The main effect of task was significant $[F(1,17)=9.07, p<$ .008]. The Task by Dimension interaction was not significant $[F(1,17)<1.00]$. Taken together, the pattern of both results indicates a symmetric pattern of interference over the two dimensions.

The pattern of reaction times illustrated in Panels $C$ and $D$, in which the discriminabilities of the stimuli were equated, indicates a mutual symmetric interference between vowel and pitch. These results are consistent with Miller's (1978) earlier findings with adults. However, she used vowels in CV syllable context rather than vowels in isolation.

The results from the final condition, consisting of a small vowel difference and a large pitch difference, are shown in Panel E. As is evident in the figure, identification in the control task when pitch was relevant was faster than it was in the control task when the vowel was relevant. A strong main effect of dimension supported this difference $[F(1,17)=$ $21.02, \mathrm{p}<.0003$ ]. Nevertheless, a mutual, symmetric pattern of interference also emerged. Specifically, performance was faster in the control than in the focusing task $[F(1,17)=12.04, p<.003]$, but this factor did not interact with the dimension that was relevant $[F(1,17)=3.13, p<.1]$.

Note the contrast between the pattern of results shown in Panels A and B and those shown in Panel E of Figure 1. When the vowels were more discriminable than the pitches, vowel identification was not affected by the variation between the pitches. In marked contrast, when the pitches were more discriminable than the vowels, pitch identification was disrupted by the small vowel variation. Does this result imply that the processing of pitch is more dependent on the processing of vowels than vowel identification is on pitch?

The answer to this question may be clarified by an examination of Figure 2. In this figure, the magnitude of interference from irrelevant variation is plotted as a function of the relative discriminability of the relevant and irrelevant dimensions. Specifically, the interference effect can be measured by calculating the difference between reaction times in the focusing task and the control task. The measure of relative discriminability was calculated by subtracting the reaction times in the control task for the irrelevant dimension from those in the control task for the relevant dimension. ${ }^{1}$

As one examines this figure, it is apparent that the vowel dimension was relatively more discriminable than the pitch dimension. This is indicated by a general rightward shift of the vowel function compared with the pitch function. Further support for this finding was obtained from pilot data, which indicated that, even with a much greater pitch difference (i.e., $135 \mathrm{~Hz}$ in the large-pitch-difference condition rather than the $75 \mathrm{~Hz}$ used in the present experiments), pitch discriminability was not increased any more than was vowel discriminability.

The critical finding is that the pattern of results displayed in this experiment does not strongly suggest an asymmetric dependency between the processing of pitch and vowel color. The effect of the magnitude of irrelevant variation relative to the relevant difference appears to be similar, both when pitch is relevant and when vowel color is relevant. In other words, there appears to be one function relating the magnitude of the interference effect to the relative discriminability of the values on the two dimensions. Which dimension is relevant does not appear to matter greatly.

Figure 2 clarifies the contrast between Panels A and $B$ and Panel $E$ shown in Figure 1. Recall that pitch identification was disrupted by vowel variation even when the discriminability of the pitches was much greater than the discriminability of the vowels, whereas vowel identification was not impaired by pitch variation when the vowels were the more highly discriminable dimension. As is evident in Figure 2, this apparent interaction between the effects of relative discriminability and the dimension relevant for classification is due to the magnitude of the differences in discriminability. In the two conditions (see Panels A and B of Figure 1) in which vowel identification was not slowed by pitch variation, the relevant vowel difference was considerably greater than the irrelevant pitch variation. However, in the condition (see Panel E of Figure 1) in which the pitch difference was the larger, this relevant difference was only somewhat larger than the irrelevant vowel difference. 


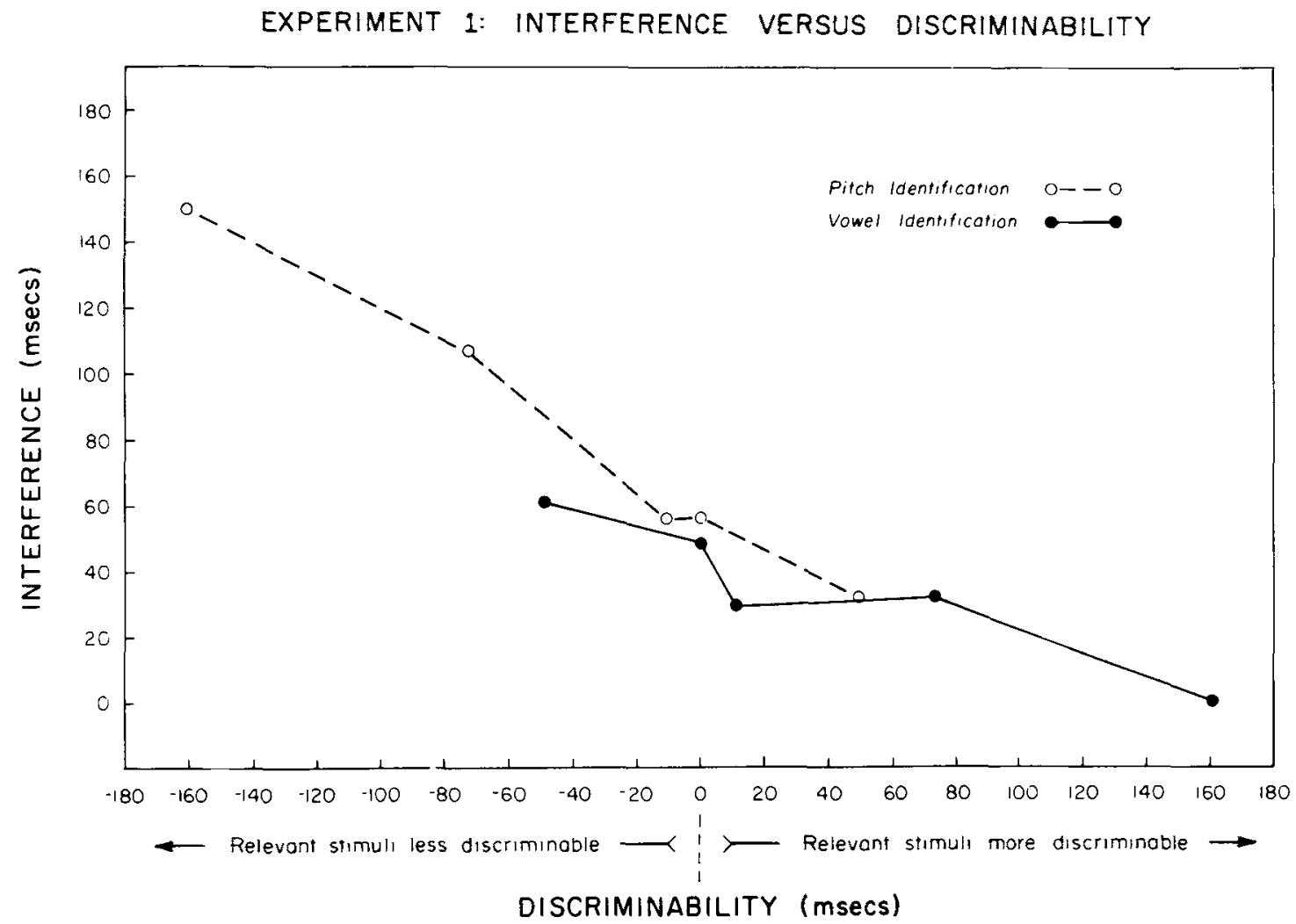

Figure 2. Interference vs. diseriminability for bolated vowels.

In summary, the findings of this first experiment do not implicate two distinct levels of processing. Rather, the identification of isolated vowels and pitch appear to be mutually dependent. Manipulations of the discriminability of the irrelevant dimension clearly affects the identification of pitch and vowel in the same way and to the same degree. This result is therefore consistent with the notion that one level of processing is involved in the identification of pitch and isolated steady-state vowels.

\section{EXPERIMENT 2}

It has often been suggested that isolated steadystate vowels are like nonspeech stimuli because, under certain conditions, they are perceived continuously rather than categorically (Liberman, 1970). When vowels are placed in dynamic phonetic contexts, they appear to be perceived more like stop consonants (Stevens, 1968). A redundant consonant (/b/) was appended to the beginning of each stimulus in Experiment 2 in order to examine the processing dependencies between vowel color and pitch when the stimuli were, perhaps, more "speech-like" because they appeared in context.

\section{Method}

Subjects. The subjects in this experiment were 102 Indiana
University students. They were paid $\$ 3 / \mathrm{h}$ or were given experimental credit for their participation. They were all native speakers of English. None of the subjects reported a previous history of a speech or hearing disorder.

Stimuli. The only difference between the stimuli in Experiment 1 and Experiment 2 was that the vowels in the second experiment were embedded in a dynamic CV syllable context. Specifically, a $/ b /$ was appended to the beginning of each stimulus so that they ranged perceptually from $\mathrm{a} / \mathrm{bi} /$ to $\mathrm{a} / \mathrm{bl} /$ to $\mathrm{a} / \mathrm{b} \varepsilon /$. There were no other differences between the stimuli used in the two experiments.

Procedure. Again, five experimental conditions were presented to five different groups of subjects. Each group of subjects participated in only one condition. The conditions may be characterized as follows: (A) large vowel difference, small pitch difference; (B) intermediate vowel difference, small pitch difference; (C) large vowel difference, large pitch difference; (D) small vowel difference, small pitch difference; and (E) small vowel difference, large pitch difference. All other aspects of the procedure and design were identical to those employed in Experiment 1.

\section{Results and Discussion}

Mean reaction times in the five conditions are shown in Figure 3 and Table 4. The data were analyzed using analyses of variance as in Experiment 1.

The large-vowel-difference/small-pitch-difference condition and the intermediate-vowel-difference/ small-pitch-difference condition (illustrated in Panels $A$ and $B$, respectively) reveal an asymmetric interference pattern in which irrelevant vowel variation interfered with pitch identification but irrelevant 
A

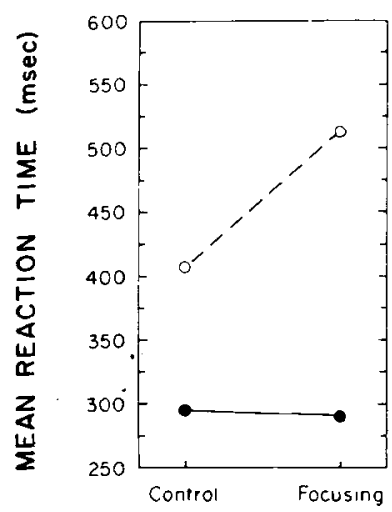

B

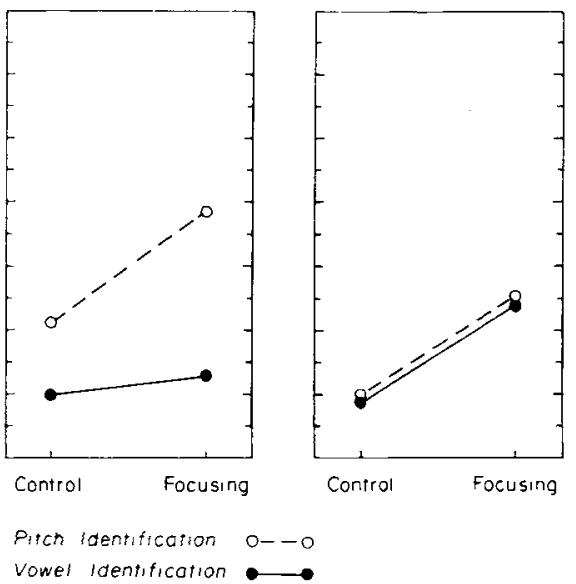

D

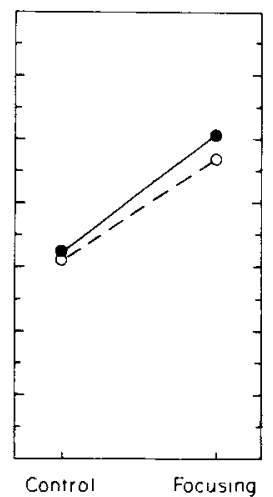

$E$

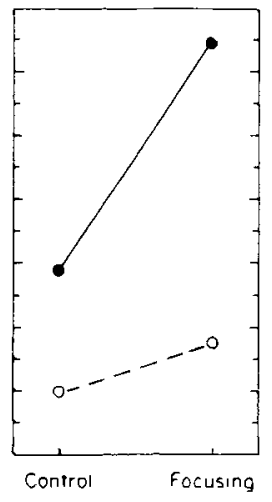

Figure 3. Mean reaction times for large-vowel/small-pitch, intermediate-vowel/small-pitch, large-vowel/large-pltch, manll-vowel/ small-pitch, and small-vowel/large-pitch conditions in Experiment 2.

pitch variation did not affect vowel identification. This conclusion is supported by the strong Task by Dimension interactions illustrated in Panel $\mathrm{A}$ $[F(1,17)=10.85, \mathrm{p}<.004]$ and in Panel $\mathrm{B}[\mathrm{F}(1,17)=$ $25.99, \mathrm{p}<.00005$ ]. In both conditions, the vowels were much more discriminable than the pitches, as shown by the highly significant main effects of dimension in both cases $[F(1,17)=54.01, p<.001$, and $F(1,17)=35.53, \mathrm{p}<.001]$.

Performance in the large-vowel-difference/largepitch-difference condition and in the small-voweldifference/small-pitch-difference condition are shown, respectively, in Panels C and D of Figure 3. The discriminabilities of the pitches and vowels do not appear to differ in these two conditions [ $t(17)<$ 1.00 and $\mathrm{t}(23)<1.00$ ]. Moreover, mutual and symmetric patterns of interference were found in both conditions, as indicated by highly significant main effects of task shown in Panels $C$ and $D[F(1,17=$ 14.58, $\mathrm{p}<.002$, and $F(1,23)=11.14, \mathrm{p}<.005]$ and by the absence of an interaction between task and irrelevant dimension in both conditions $[\mathrm{F}(1,17)=$ .004 and $F(1,23)=.13$ ]. Thus, identification of the values on either dimension was slowed down by irrelevant variation on the other dimension.
Panel E illustrates the results in the small-voweldifference/large-pitch-difference condition, a condition in which the pitches were now more discriminable than the vowels. In this condition, an asymmetric interference pattern emerged $[F(1,17)=7.16$, $p<.01$ ] that was the opposite of that shown in Panels $A$ and $B$.

The results across all five conditions in Experiment 2 display a consistent pattern. When the values on the two dimensions did not differ in discriminability, mutual and symmetric interference was observed. When the values on one dimension were more discriminable than they were on the other, a pattern of asymmetric interference was observed. The dimension with the largest variation interfered with judgments of the dimension with the least variation. However, the dimension containing less variation did not interfere with judgments of the dimension on which variation was greatest.

This analysis, however, is incomplete. Figure 4 illustrates the magnitude of the interference effect as a function of the relative discriminability of the values on the relevant and irrelevant dimensions. As is evident in the figure, changes in the magnitude of irrelevant pitch variation affect vowel identification

Table 4

Mean Reaction Times (in Milliseconds) for All Conditions in Experiment 2

\begin{tabular}{|c|c|c|c|c|c|c|c|c|c|c|}
\hline & \multicolumn{2}{|c|}{$\begin{array}{l}\text { Large Vowel, } \\
\text { Small Pitch }\end{array}$} & \multicolumn{2}{|c|}{$\begin{array}{l}\text { Intermediate Vowel, } \\
\text { Small Pitch }\end{array}$} & \multicolumn{2}{|c|}{$\begin{array}{c}\text { Large Vowel, } \\
\text { Large Pitch }\end{array}$} & \multicolumn{2}{|c|}{$\begin{array}{l}\text { Small Vowel, } \\
\text { Small Pitch }\end{array}$} & \multicolumn{2}{|c|}{$\begin{array}{l}\text { Small Vowel, } \\
\text { Large Pitch }\end{array}$} \\
\hline & $\mathbf{P}$ & $\mathbf{V}$ & $\mathbf{P}$ & $\mathbf{V}$ & $\mathbf{P}$ & $\mathbf{V}$ & $\mathbf{P}$ & $\mathbf{V}$ & $\mathbf{P}$ & $\mathrm{V}$ \\
\hline $\begin{array}{l}\text { Control } \\
\text { Focusing }\end{array}$ & $\begin{array}{l}407 \\
513\end{array}$ & $\begin{array}{l}290 \\
278\end{array}$ & $\begin{array}{l}356 \\
443\end{array}$ & $\begin{array}{l}299 \\
314\end{array}$ & $\begin{array}{l}299 \\
376\end{array}$ & $\begin{array}{l}293 \\
368\end{array}$ & $\begin{array}{l}404 \\
483\end{array}$ & $\begin{array}{l}411 \\
502\end{array}$ & $\begin{array}{l}298 \\
335\end{array}$ & $\begin{array}{l}393 \\
571\end{array}$ \\
\hline
\end{tabular}

Note $-P=$ pitch $I D ; V=$ vowel $I D$. 


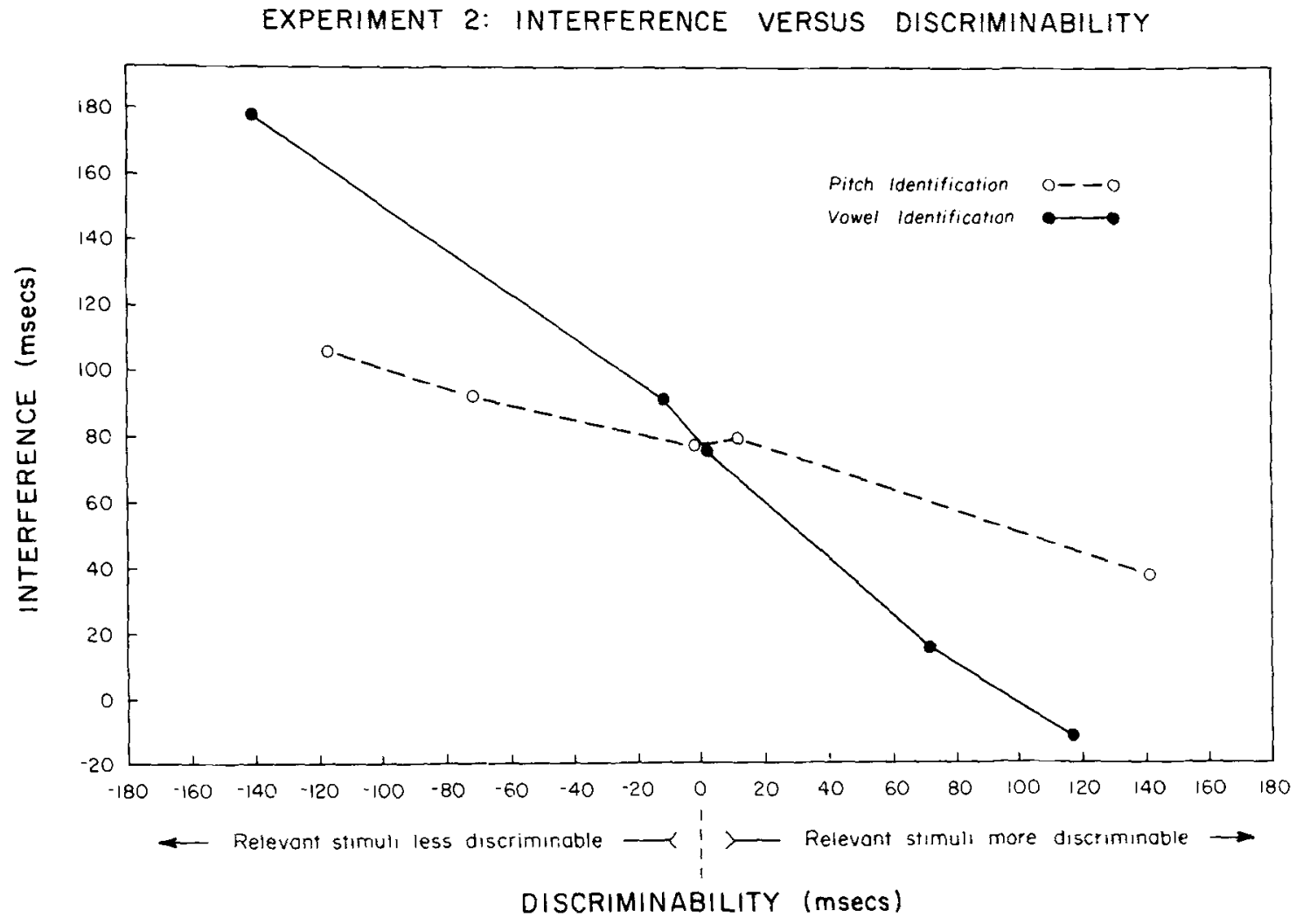

Figure 4. Interference vs. discriminablity for vowels in $₫ \mathrm{CV}$ context.

much more than changes in the magnitude of irrelevant vowel variation affect pitch identification. This is reflected by the steeper slope of the function when vowel is the relevant dimension compared with when pitch is the relevant dimension. This pattern of results strongly supports the hypothesis of two levels of processing-a general auditory level of analysis that precedes a more specific phonetic level. Simply put, manipulations of a general auditory dimension affect decisions about speech more than do manipulations of a phonetic dimension affect decisions about a dimension characteristic of sounds in general.

The pattern of results obtained in this experiment therefore contrasts with those obtained in Experiment 1 . Clearly, vowels in the context of a consonant are processed differently from the way in which vowels in isolation are. Specifically, vowels appear to be processed at a more phonetic level when in the context of a consonant than when presented in isolation. Another specific comparison between the results of the two experiments supports the claim that vowels are processed differently in different contexts. In contrast with Experiment 1 , the ranges of discriminability of the vowels and pitches were very closely matched in this second experiment. The vowel and pitch functions overlap considerably more in Figure 4 than they do in Figure 2. Moreover, the discriminability of the vowels decreased when they were preceded by a consonant as opposed to when they were presented in isolation.

In summary, the results of this experiment provide additional support for the existence of a phonetic level of processing that follows and depends on processing at a more general auditory level of analysis. When vowels are embedded in a CV syllable, they appear to be processed at this second level.

\section{GENERAL DISCUSSION}

Three major results emerged from these experiments. First, the results provide additional evidence for two stages of processing in the perception of speech. Second, the results support the notion that vowels are processed by the general auditory system when presented in isolation and by a secondary phonetic-like system when presented in the context of a consonant. Third, the results demonstrate the importance of examining processing dependencies between stimulus dimensions across ranges of specific stimulus values.

The first result is important because a two-stage model of speech processing is generally assumed (e.g., Foss \& Blank, 1980; Pisoni, 1978; StuddertKennedy, 1976), but support for such a global difference-one that is characteristic of the processing of both consonants and vowels-has been mixed (e.g., Ganong, 1979; Klatt, 1979; Miller, 1978). The second 
result is also consistent with earlier conclusions about the processing of vowels. Specifically, vowels appear to be more "speech-like" when placed in normal phonetic contexts than when appearing in isolation (e.g., Godfrey, 1974; Stevens, 1968).

It is the third point-the methodological one-that may be the most significant contribution of the present studies. Note that if we had merely equated the discriminability of the two dimensions and not investigated their interaction across a range of discriminabilities, as previous investigators have done (Miller, 1978; Wood, 1975), our conclusions would have been quite different. Specifically, the findings when the two dimensions do not differ in discriminability imply mutual and symmetric dependencies in the processing of vowel color and pitch. Moreover, such an effect emerges both when the vowels were presented in isolation and when they were preceded by a stop consonant. Like Miller (1978), we would have been led to conclude that the dependency between the processing of vowels and pitch was different from that observed by Wood (1975) for the processing of stop consonants and pitch. In addition, the results would have suggested that the dependency between the processing of vowels and pitch did not depend on the context in which the vowel was presented. Neither of these conclusions gains support from the present analysis. Rather, the processing of vowels, at least in a speech-like context, depends more on variations in a general auditory dimension than the processing of pitch depends on variations in vowel color. This result emerged only when we considered identification performance over a wider range of discriminabilities than typically used in past studies.

The observed interaction between the processing of vowels in the context of a consonant and the processing of pitch thus appears to be rather like the interaction, first reported by Wood (1975), between the processing of consonants and pitch. In both cases, the identification of a phoneme is affected more by pitch variation than is identification of pitches by variation of the phonemes. The only difference in the precise nature of the interaction may be a relatively trivial one. Specifically, when the discriminabilities of the pitches and vowels do not differ, interference effects of equal magnitude are observed when vowel color is relevant and when pitch is relevant. Wood's (1975) findings would suggest, however, that when the discriminabilities of the pitches and consonants do not differ, the interference effect should be greater when vowel color is the relevant dimension than when pitch is the relevant dimension. In light of the present analysis, this apparent difference in the interaction of vowel color and pitch and of consonant variation and pitch may not be important. The effects of differences in relative discriminability of the pitches and phonemes may be the same whether the phoneme is a consonant or a vowel. The only differ- ence may be the point at which the two functionspitch identification as a function of the magnitude of the vowel difference and vowel identification as a function of the magnitude of the pitch differenceintersect. This point may not be a critical one for understanding the nature of the underlying processing. The more important issue is whether the two functions are the same or different because different functions implicate asymmetric interactions in the processing of speech and nonspeech signals.

In conclusion, the present experiments provide additional support for the claim that speech perception involves two stages of processing-a general auditory analysis followed by a more specific processing of information relevant to the classification of speech sounds. Furthermore, the identification of vowels in consonantal context results from processing of the acoustic signal at both stages, whereas the identification of isolated vowels appears to involve only the first stage. Finally, these conclusions stem from an examination of the interaction between the processing of vowels and pitches across a relatively wide range of specific vowel and pitch differences. An asymmetric pattern of interaction should be evident as a function of changes in the specific values on the two dimensions if such asymmetries are due to levels of processing rather than to only the interaction of specific values or specific dimensions of stimuli.

\section{REFERENCE NOTES}

1. Ganong, W. F. The internal structure of consonants in speech perception: Acoustic cues, not distinctive features. Unpublished manuscript, June 1979.

2. Kewley-Port, D. KLTEXC: Executive program to implement the KLATT software speech synthesizer (Research on Speech Perception, Progress Report No. 4). Bloomington: Indiana University, 1978.

\section{REFERENCES}

Crowder, R. G. The sound of vowels and consonants in immediate memory. Journal of Verbal Learning and Verbal Behavior, 1971, 10, 587-596.

Crowden, R. G. Precategorical acoustic storage for vowels of short and long duration. Perception \& Psychophysics, 1973, 13, 502-506. (a)

Crowder, R. G. Representation of speech sounds in precategorical acoustic storage. Journal of Experimental Psychology. 1973, 98, 14-24. (b)

DAY, R. S., \& Woon, C. C. Mutual interference between two linguistic dimensions of the same stimuli. Journal of the Acoustical Society of America, 1972, 52, 175. (Abstract)

Foss, D. J., \& Blank, M. A. Identifying the speech codes. Cognitive Psychology, 1980, 12, 1-31.

Garnfr, W. R., \& Morton, J. Perceptual independence: Definitions, models, and experimental paradigms. Psychological Bulletin, 1969, 72, 233-259.

Gonfrfy, J. Perceptual difficulty and the right ear advantage for vowels. Brain \& Language, 1974, 1, 323-335.

Kr.ATr, D. H. Speech perception: A model of acoustic-phonetic analysis and lexical access. Journal of Phonetics, 1979, 7 , 279-312. 
KUнt, P. K. Speech perception in early infancy: Perceptual constancy for vowel categories. Journal of the Acoustical Society of America, 1975, 60, S90.

KUн1, P. K. Speech perception in early infancy: The acquisition of speech-sound categories. In S. K. Hirsh, D. H. Eldridge, I. J. Hirsh, \& S. R. Silverman (Eds.), Hearing and Davis: Essays honoring Hollowell Davis. St. Louis, Mo: Washington University Press, 1976.

Kuhl, P. K., \& MilleR, J. D. Speech perception in early infancy: Discrimination of speech-sound categories. Journal of the Acoustical Society of America, 1975, 58, S56.

Liberman, A. M. Some characteristics of perception in the speech mode. In D. A. Hamburg (Ed.), Perception and its disorders, Proceedings of A.R.N.M.D. Baltimore: Williams and Wilkins, 1970.

Mrt...., J. L. Interactions in processing segmental and suprasegmental features of speech. Perception \& Psychophysics, $1978,24,175-180$.

Pisoni, D. B. On the nature of categorical perception of speech sounds. Unpublished doctoral dissertation, University of Michigan, 1971.

PISON, D. B. Auditory and phonetic memory codes in the discrimination of consonants and vowels. Perception \& Psychophysics, 1973, 13, 253-260.

Pisoni, D. B. Auditory short-term memory and vowel perception. Memory \& Cognition, 1975, 3, 7-18.

Pisoni, D. B. Speech perception. In W. K. Estes (Ed.), Handbook of learning and cognitive processes (Vol. 6). Hillsdale, N.J: Erlbaum, 1978.

STEVENS, K. N. On the relations belween speech movements and speech perception. Zeitschrift für Phonetik, Sprachwissenschaft und Kommunikationsforschung, 1968, 21, 102-106.
Studdert-Kennedy, M. Speech perception. In N. J. Lass (Ed.), Contemporary issues in experimental phonetics. Springfield, Ill: Thomas, 1976.

Wood, C. C. Auditory and phonetic levels of processing in speech perception: Neurophysiological and information processing analysis. Journal of Experimental Psychology: Human Perception and Performance, 1975, 104, 1-33.

Wood, C. C., Goff, W. R., \& DAY, R. S. Auditory evoked potentials during speech perception. Science, 1971, 173, 12481251

\section{NOTE}

1. Because of the nature of these two measures, Figure 1 is isomorphic to a figure in which performance in the focusing task is displayed as a function of performance in the control task for the dimension that is irrelevant in the focusing task. In other words, Figure 2 (and Figure 4) displays performance in a focusing task as a function of the discriminability of the irrelevant dimension. Rather than simply using performance in the focusing task for one dimension and performance in the control task for the other, we used the present two measures of the magnitude of the interference effect and of the discriminability of the relevant and irrelevant dimensions. We chose to do this because the first two measures are simpler to discuss in a way that is pertinent to the present phrasing of the hypothesis under consideration.

(Received for publication April 7, 1980; accepted revision received October 25,1980 .) 